\title{
Design of Geographic Information System in Da Qing Oil Field Gas Pipe Network
}

\author{
Yang Shuren ${ }^{\mathrm{a}}$, Peng Peng ${ }^{\mathrm{b}}$ \\ ${ }^{a}$ Petroleum Engineering College, Northeast Petroleum University, Daqing , China \\ ${ }^{b}$ Petroleum Engineering College, Northeast Petroleum University, Daqing , China
}

\begin{abstract}
Combined with the actual requirements of gas pipe network management, based on the analysis of significance and effect on establishing geographic information system in Daqing nature-gas company, overall design of geographic information system in Daqing oil field gas pipe network, network structure and the analysis of spatial data and the attribute data are accomplished, what's more, graphic and attribute data is unified to store, process and analyse, using geographic information system as technical support, at the same time, the spatial database is established, which is for the maintenance and planning of gas pipe network, piping design, pipeline construction, the completion of information, the repairing decision, as well as, inquiring the relative attribute data. This paper introduces the data and network structure, in addition to, security of geographic information system in Daqing oil field gas pipe network specifically, the system chooses MS Windows 2000 (Advanced) Server or UNIX as operating system, furthermore, using other geographic information system softwares to build geographic information development platform, giving priority to the ArcGIS of ESRI company. The result shows that the development of project has reached the expected goal, laying the solid foundation for system subsequent development and further researching work.
\end{abstract}

Index Terms: Gas pipe network; Geographic information system; Data structure; Network structure; Environment configuration; Principle; Security

(C) 2011 Published by MECS Publisher. Selection and/or peer review under responsibility of the International Conference on E-Business System and Education Technology

\section{Introduction}

People attach importance to the information increasingly, which is the rapid development of the industry, the information revolution wave[1] is impacting the human society. In this revolution, geographic information system is growing up rapidly and developing that sets computer science, geography, surveying and mapping, remote sensing, environmental science, urban science, space science, information science and scientific management a new frontier science, using GIS technique to build the gas pipe network information system, which is a graphics database management system, that maps urban topographic map, stacks gas network diagram

Corresponding author:

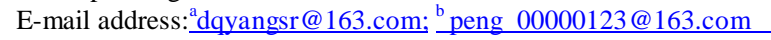


and associates the corresponding pipe and equipment with graphics. The system assists management personnel in all to file management, maintenance and handle the sudden accident from the dot, line, face on the electronic map[2], that has the traditional file management incomparable advantages of accurate and fast.

With the continuous expansion of the size of oildom and Daqing and the increasingly improvement of enterprise modernization, digitization and science degree, the gas pipe network architecture of Daqing oilfield gas limited liability company will be more and more intensive, complicated and there will be more frequent updates, in addition, wovened with the water supply, the power supply and other pipe network together, different laying of times and construction standards, all kinds of pipeline accidents, such as gas interruption, leakage and burst pipe sometimes happen as well. The job pressure of network reconstruction, pipeline leak repair has a strong impact on the life and work order of urban residents and oildom. In order to adapt to the development of Daqing oilfield gas industry and improve the modern management level, it is imperative to establish and make full use of pipe network geographical information system, which can provide various professional required information for assignments, such as, gas planning, design, construction, safety supply, production scheduling, equipment maintenance, pipeline network transformation and rescue and so on.

\section{The general structure}

According to the establishment principles of geographic information system in Daqing oilfield gas pipe network, the construction of the system and the functional requirements are closely related to the development goal the system, the specific goals should be built based on scientific and reasonable user demand analysis. Therefore, different cities and departments have certain differences on the construction and function of system, generally speaking, the urban pipe network geographic information system should have the following basic functions: data acquisition, editorial changes, storage administration, inquiry statistics, information output, analysis and operation of pipe network, three-dimensional network generation, subsystem or functional module.

\subsection{Data Structure of System}

\subsubsection{Basic topographical map spatial databases.}

The application can't do without the support of basic topographical map, which not only is the the background picture of pipe network, but also provides terrain reference for profile analysis of pipe network. Therefore, while establishing the geographical information system of pipe network, basic topographical map spatial databases should be built, which should contain topographic maps and attributes information.

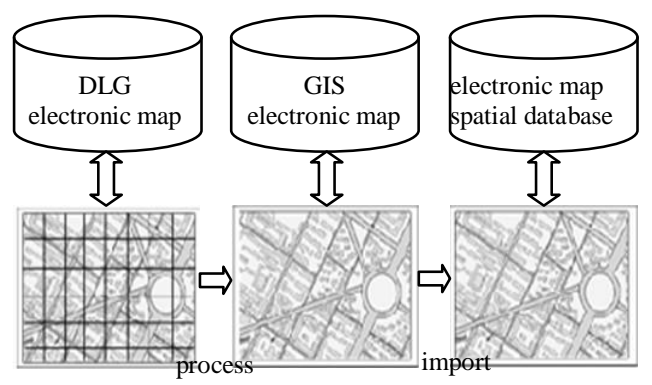

Fig 1. Basic topographical map spatial databases creation process. 
Electronic map spatial database mostly contain vector data (basic topographic maps data, part of the special current information extracted by basic topographic maps, grade control point data) and raster data[3] (the important products of digital surveying and mapping production, there are lots of advantages of intuitive, informative, and readable).

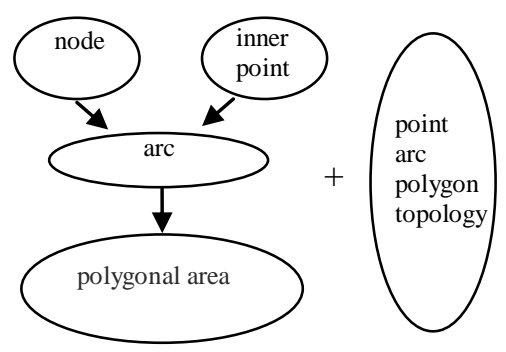

Fig 2. Vector data model.

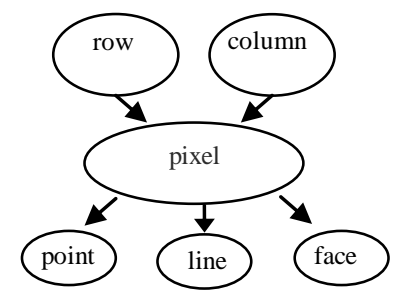

Fig 3. Raster data model.

\subsubsection{Gas pipe network spatial databases.}

Aiming at the different information source, different methods to obtain and deal with the data must be adopted, the technology those are often used including: collection of field data, which plays the characteristic of terrestrial digital mapping is flexible and easy to modify, having local measurement and real-time update, so as to keep topographic maps "fresh" all along; the original drawing (base map) data acquisition, which is divided into manual digital input, the scanned vectorization and tie point graph; data conversion format: processing the existing gas data network data of electronic format to generate pipe network database of geography that meets the requirements of geographic information system.

\subsubsection{Geographic information base management based on Client/Server.}

Client/Server, which not only is main stream architecture of modern computer system, but also lowcost and open. The management of geographic information base, with the Client/Server for benchmark model, is divided into two levels: (1) using DBMS as the main part of database management platform of geographic information system[4] (including spatial information and related attributes information), however, geographic information is integrated into the RDBMS through spatial database engineering (ArcSDE 9.x), (2) geographic information database server is constituted by DBMS, moreover, using all kinds of application softwares, which are developed by ARCINFO 9.x or ARCVIEW 9.x as client, that is Client/Server. 


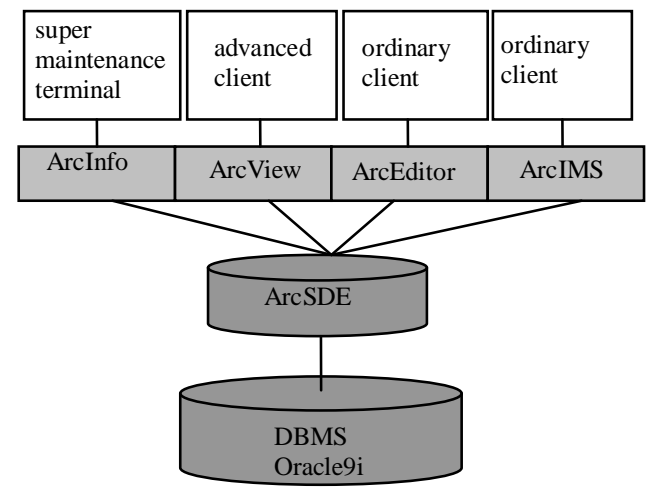

Fig 4. The management of database

\subsection{Network Structure of System}

\subsubsection{Network topology.}

The system will constitute the wide-area network in the city, which is going to form the central application of gas geographic information in Daqing, so that it can connect every application sever and local area network with gas geographic information base to interact and data transmission, whose reliability and function play an important role of all the system. The network topology of system is a centralized network topology with a physical layout that resembles a star. the backbone network puts to use the routing fabric, the communication protocol of backbone network is TCP/IP, and communication medium is the fiber network.

\subsubsection{The dynamic data replication.}

In order to guarantee the security of data system, database snapshot of Oracle is put to use, backing up the system data at regular time. Using database snapshot to do the dynamic data replication, it can specify the time cycle of data updated and the reproduction process of database automatic management.

\subsection{System Security}

\subsubsection{System security of hosts.}

To provide user confirmation and check permissions for login, which is used for prohibiting illegal user from using computer. For the prevention from infection and destruction of viruses and worms, users check the system with special software and upgrade it at regular intervals (or using virus-fire wall and platform).

\subsubsection{Data security.}

Data security refers to mainly consistency and integrality of the spatial data. It has access an efficiency management of system privilege by the means of security management subsystem, at the same time, doing the check permissions user log in, according to the function of the authority open and shielding system, prevent unauthorized users modifying and destructing the data by system operation. In order to avoid unauthorized users to modify attribute data[5] of system, it is important to set user privilege control, system administrator backup the database and share files at fixed period to prevent the destruction of hardware damaging data security. Using the relevant functions of Oracle to backup the database. 


\subsubsection{Network security.}

In order to prevent the infection and destruction of viruses and worms, in addition, lawless users in the network are likely to threaten on the network security seriously at any moment, it is must to make use of the technology of software and hardware firewall, limiting the specific Internet users access system using security mechanism built-in IMS.

\section{The system environment configuration}

The application of geographical information system has to store plenty of map data, while selecting and processing map and do lots of calculation and transformation. Therefore, it requires a high degree of ability of operating rate, storing capacity and graphic processing.

\subsection{Software Environment of System}

\subsubsection{Operation system platform.}

Server operating system platform: using high-quality computer or minicomputer as master server and MS Windows 2000 (Advanced) Server or UNIX as operating system; client operating system platform: using Windows 2000 Professional to operate the application of ArcInfo 9.x.

\subsubsection{Geographic information system platform.}

Using other geographic information system software to build geographic information development platform and giving priority to the ArcGIS of ESRI company, the system supporting platform software of geographic information in ArcGIS software of ESRI company mainly, including ArcInfo 9.x, ArcSDE 9.x, ArcIMS, ArcGIS Engine9, MrSid.

\subsubsection{Data base management system.}

This system makes use of Oracle $9 i$ as the database platform of all application system. Oracle and ArcSDE 9.x are the core of the solution to establish enterprise level geographic information system.

\subsubsection{The development platform.}

The system chooses VB $6.0+\mathrm{VC}++6.0$ as the main development platform of system.

\subsection{Hardware Environment of System}

\subsubsection{The system server.}

Database server, Web publish server and the primary domain control server are all needed. In order to keep hardware of system compatibility and consistency, considering the hardware maintenance and security of system, it is better to use IBM as system server.

\subsubsection{Client.}

Client is applied to input and inquire the data, which is the user interactive interface to get geographic information from database, it meets the requirements to use current middle and top grade computer. The specialized geographic information system client should make use of high-end computer, owing to collect, store and manage the spatial data, as well as the complicated data modeling and analysis. 


\subsection{Network Environment of System}

\subsubsection{Wide area network.}

WAN is made up of backbone[6] network, the high speed communication network and the routes. Backbone network connect server with all LAN, using FDDI double-loop fiber network can meet the requirements of UBGIS. Configurating the remote router and using the high speed communication network, which is applied for posts and telecommunications network, connect LAN of each unit with backbone network separately.

\subsubsection{Local area network.}

Ordinary or fast Ethernet is used to meet the requirements of operation service.

\subsubsection{Transmission control protocol/Internet protocol}

Using TCP/IP, there are some characteristics as follows: network technology is independent and independence of the hardware of any manufacturer; connect capability is strong, any two computers can exchange the information in the Internet; supporting the standard protocol for applications, including email, file transfer, remote registry and so on.

\section{The normalization and standardization of system}

The most important characteristic of GIS is to keep lots of information from each department and major unified management, according to the certain standards and norms, so as to have a data base of standardization and normalization, moreover, it can achieve the standardization and automation of the government office by means of the standardized operation procedures from information system.

\subsection{Principles of Normalization and Standardization}

Coding scheme should apply for the collection, storage, retrieval, analysis, output and exchange of topographical elements with the large-scale urban digital topographic maps and the production of pipe networks graph, as well as the establishment of all kinds of spatial information[7] system; item, meaning and symbol configuration of network elements should obey the rule of national or industry standards; be appropriate for the application and management of modern computer and database technology and the purpose of digital cartography, according to the attributes and characteristics of topographical elements, make the scientific classification to form the classification scheme of system; code structure of project keep appropriate allowing for expansion; not limiting the map scale of pipe network, the same element has the only classification code with variable scales of map library; the planning has stronger portability and easy to exchange with the different coding schemes.

\subsection{Principles of Network Coding}

At present, the code of burning pipeline and underground facilities points in Daqing is mainly blank, the comparision between graph and card is so difficult, which leads to the great inconvenience to file management, equipment maintenance, the management of rushing to repair, scheduling and operating. Therefore, coding to the pipeline of the achievement of field completion measurement in a unified way becomes so important, which can ensure the unique identification code of system.

\subsubsection{Coding of pipe section (numeric type).}

A pipeline is made up of multi-step pipe sections on the each road, pipe section is a section of pipeline to analyse the pipe network for convenience, which can use tee joint point, four-way point, metamorphic point or 
variable diameter point as the crossover points of pipe section. Underground pipe network generally is buried along the road, so it can use the road code as alignment code, the structure of coding as follows (ten digit):

$\begin{array}{ccl}\text { localization and division code } & \text { direction code } & \text { road code } \\ \text { XX } & \mathrm{X} & \mathrm{XXX} \\ \text { coding of pipeline } & \text { serial number } & \\ \text { X } & \mathrm{XX} & \end{array}$

4.2.2 Coding of pipe points (character type).

$\begin{array}{cc}\text { 5000 map sheet code } & 2000 \text { map sheet code } \\ \text { XXXX } & \text { X } \\ \text { 1000 map sheet code } & \text { 500 map sheet code } \\ \text { X } & \text { X } \\ \text { 5000 map sheet code } & \text { 2000 map sheet code } \\ \text { XXXX } & \text { X } \\ \text { type } & \text { serial number } \\ \text { X } & \text { XXX }\end{array}$

The detailed operational requirements as follows: the two former show the code of 1:5000 topographic maps concordance list; 2000 map sheet code, such as: 1, 2, 3, 4; which is the same as 1000 and 500 map sheet code; type code: $\mathrm{T}$ is pressure regulating station, $\mathrm{S}$ is well, $\mathrm{F}$ is valve well, $\mathrm{J}$ is node, $\mathrm{G}$ stands for tank station, the serial number is from 001 to 999 according to the sequential arrangement, from north to south or from west to east.

\subsubsection{Numbers of the valves (character type).}

In the south: from XX Road to XX Bridge for boundary, the writing direction is from east to west or from north to south; in the north: the boundary is the same as above, but the writing direction is from south to north; the trunk pipe: using the road name to fill in; the branch pipe: it is divided into left and right, that is, writing odd numbers on people's left hand side, and the other side to write even numbers; the valves of adding midway: the valve which is between two valves is written "the newly increased valve".

\section{Conclusions}

According to system architecture and the characteristics of gas pipe network data, the analysis of spatial data and attribute data is finished, so as to achieve the design of database and software implementation. If the user has the new requirements after operating the system, the module can be developed to connect the original system, which shows that the system owns the better expandability.

Based on the analysis of the data characteristics and spatial data storage of gas pipe network, a strategy of image transmission is given to be suitable for Web. Preprocessing the image, grid registration, the feasible registration strategy can be proposed according to the actual situation and get the quintessence from others.

The development of urban gas geographic information system is a lengthy process, which needs the mutual efforts from the program developer and gas technicist, so as to enable the system function to consummate unceasingly. 


\section{References}

[1] LI Fan, ZHANG Yang. Design of urban gas pipe network geographic information system [J]. Shanghai Gas Journal vol. 247, pp. 1-3, June 2005. (in Chinese)

[2] LV Hai-bo, YANG Wei-hua.. Research of Feng Feng gas pipe network geographic information system based on GIS [J]. Science and Technology Consulting Herald Journal vol. 2., October 2007, pp.67-69. (in Chinese)

[3] LIU Xiao-jie. A comparison of vector and gridded data pattern by GIS [J]. Jilin Geology Journal vol. 24 No.1 March 2005, pp. 89-91. (in Chinese)

[4] TIAN Yi-mei, ZHAO Xin-hua. The application and development of GIS in water supply system [J]. China Water \&Wastewater Journal. vol. 16 No.9 March 2000, pp. 57-62. (in Chinese)

[5] CHEN Shi-xian, WANG Yuan-zhen. Establishment of the geographic information system in Wisco Daye iron water supply pipe network [J]. Geotechnical Engineering World vol. 3 No.7 2000, pp. 41-45. (in Chinese)

[6] ZHANG Wen-jun. Establishment of natural gas pipe network system based on MAPGIS [J]. Earth Science China Studies Juornal vol. 27 No.3 2002, pp. 250-254. (in Chinese)

[7] P.A.Burrough. Principles of GIS and Resource.Oxford: Clarendon Press,1986, pp. 107-151. 